\title{
CANONICAL COORDINATES AT A POINT FOR TWO SKEW-SYMMETRIC TENSORS
}

\author{
G. H. F. GARDNER
}

1. Introduction. The problem to be considered concerns an algebraic property of two skew-symmetric tensors at a point. Let $P_{r s}$ and $Q_{r s}$ be two skew-symmetric tensors. Under a transformation $C_{s^{\prime}}^{r}$ $=\partial x^{r} / \partial x^{s^{\prime}}$ they become, in the new coordinate system, $P_{r^{\prime} \varepsilon^{\prime}}$ and $Q_{r^{\prime} s^{\prime}}$, where

$$
P_{r^{\prime} s^{\prime}}=C_{r^{\prime}}^{m} C_{8^{\prime}}^{n} P_{m n}, \quad Q_{r^{\prime} s^{\prime}}=C_{r^{\prime}}^{m} C_{s^{\prime}}^{n} P_{m n}
$$

We wish to find conditions on $P_{r s}$ and $Q_{r s}$ under which there will exist a nonsingular transformation $C_{s^{\prime}}^{r}$ which does not reorient the axes, such that

$$
\begin{array}{lll}
P_{1^{\prime} 2^{\prime}}=Q_{3^{\prime} 4^{\prime}}, & Q_{1^{\prime} 2^{\prime}}=-P_{3^{\prime} 4^{\prime}}, & P_{2^{\prime} 3^{\prime}}=Q_{1^{\prime} 4^{\prime}}, \\
Q_{2^{\prime} 3^{\prime}}=-P_{1^{\prime} 4^{\prime}}, & P_{3^{\prime} 1^{\prime}}=Q_{2^{\prime} 4^{\prime}}, & Q_{3^{\prime} 1^{\prime}}=-P_{2^{\prime} 4^{\prime}}
\end{array}
$$

at a point $A$. In view of the restrictions on the transformation it is necessary that

$$
\operatorname{det} C_{s^{\prime}}^{r}>0 \text {. }
$$

2. Let $\eta^{\text {rs }}$ denote the diagonal array $(1,1,1,-1)$ and $\epsilon^{\text {rstu }}$ the permutation symbol on 1234 . We may write equations (1.2) as

$$
\eta^{r m} \eta^{s n} P_{r^{\prime} \varepsilon^{\prime}}=\epsilon^{m n t u} Q_{t^{\prime} u^{\prime}}
$$

This is equivalent to the equation

$$
\eta^{r m} \eta^{s n} Q_{r^{\prime} s^{\prime}}=-\epsilon^{m n t u} P_{t^{\prime} u^{\prime}}
$$

using latin indices ranging from 1 to 4 with the summation convention throughout.

A coordinate system in which (2.1) and (2.2) hold, we shall call a $P$-canonical coordinate system. If we interchange $P_{r^{\prime} s^{\prime}}$ and $Q_{r^{\prime} s^{\prime}}$ in (2.1) or (2.2) we get a $Q$-canonical coordinate system. We shall prove later that a $P$-system can be obtained from a $Q$-system by a transformation restricted as in (1.3) and vice-versa. For convenience we may define

$$
(\epsilon P Q)=1 / 2 \epsilon^{m n t u} P_{m n} Q_{t u} .
$$

In this notation it is useful to note that

Received by the editors July 5, 1948 and, in revised form, February 1, 1950. 


$$
\operatorname{det} P_{r s}=(\epsilon P P)^{2}
$$

and also

$$
\epsilon^{m n t u} P_{m n} Q_{u s}=1 / 2 \delta_{s}^{t}(\epsilon P Q) .
$$

Here we may dispose of some trivial cases. If at the point we are considering one of the tensors should happen to be identically zero, then the other must also be identically zero if canonical coordinates are to exist. This is clear from equation (2.1). If the two tensors should happen to be linearly dependent at the point, then both tensors must be identically zero, if canonical coordinates are to exist. For in such a system we would have

$$
a P_{r^{\prime} s^{\prime}}+b Q_{r^{\prime} s^{\prime}}=0
$$

multiply this by $b \eta^{r m} \eta^{s n}$,

$$
\begin{aligned}
b^{2} \eta^{r m} \eta^{s n} Q_{r^{\prime} s^{\prime}} & =-a b \eta^{r m} \eta^{s n} P_{r^{\prime} \varepsilon^{\prime}} \\
& =-a b \epsilon^{m n t u} Q_{t^{\prime} u^{\prime}} \\
& =+a^{2} \epsilon^{m n t u} P_{t^{\prime} u^{\prime}} \\
& =-a^{2} \eta^{r m} \eta^{s n} Q_{r^{\prime} s^{\prime}}
\end{aligned}
$$

Therefore we would have $Q_{r^{\prime} s^{\prime}} \equiv 0$ and similarly $P_{r^{\prime} s^{\prime}} \equiv 0$.

3. The following three theorems suffice to solve the problem.

Theorem I. If $p_{r s}$ and $q_{r s}$ are two linearly independent, skewsymmetric tensors with matrices of rank 2 at a point $A$, then there exists a transformation of positive Jacobian to coordinates such that at the point $A$ all six components of each tensor vanish except:

(i) one in $p_{r s}$ and one in $q_{r s}$, if $(\epsilon p q) \neq 0$,

(ii) two in $p_{r s}$ and two in $q_{r s}$, if $(\epsilon p q)=0$.

THEOREM II. If $p_{r s}$ and $q_{r s}$ are two linearly independent, skewsymmetric tensors, with matrices of rank 2 at a point $A$, then both $p$ canonical and q-canonical coordinates can always be found for them at the point $A$ by means of a transformation with a positive determinant.

THEOREM III. If $P_{r s}$ and $Q_{r s}$ are two linearly independent, skew-symmetric tensors, then a necessary and sufficient condition that both $P$-canonical and Q-canonical coordinates may be found at a point $A$ by means of a transformation with a positive determinant is that, at $A$,

$$
(\epsilon P P)+(\epsilon Q Q)=0 .
$$

Proof of Theorem I. The assumption that the two tensors are of 
rank 2 is equivalent to assuming that the determinant of each vanishes and that neither tensor is identically zero, ${ }^{1}$ since the tensors are of rank two and equations

$$
p_{r s} \lambda_{a}^{s}=0(a=1,2), \quad q_{r s} \lambda_{b}^{s}=0 \quad(b=3,4)
$$

can be satisfied by real values of $\lambda_{(a)}^{s}$ and $\lambda_{(b)}^{s}$. The vectors $\lambda_{(1)}^{s}$ and $\lambda_{(2)}^{s}$ are linearly independent and arbitrary within a linear homogeneous combination. For example $\lambda_{(1)}^{r}=B_{1 m} \epsilon^{m r t u} p_{t u}$ and $\lambda_{(2)}^{r}=B_{2 m} \epsilon^{m r t u} p_{t u}$ are solutions where $B_{1 m}$ and $B_{2 m}$ are any linearly independent sets of constants. This may be seen by using (2.4). $\lambda_{(a)}^{r}$ thus defines a 2 -flat at the point $A$ which we shall call the $p$-plane. Similarly $\lambda_{(b)}^{r}$ defines a 2-flat at the point $A$ which we shall call the $q$-plane. These two planes will intersect in a common line if and only if (3.2) can be satisfied by a common vector $\lambda^{r}$. For this it is necessary and sufficient that det $\left(p_{r s}-k q_{r s}\right)=\left(\epsilon p p-2 k \epsilon p q+k^{2} \epsilon q q\right)^{2}=0$ for all $k$, that is,

$$
(\epsilon p q)=0 \text {, }
$$

since $(\epsilon P p)=(\epsilon q q)=0$, and $k$ is arbitrary. Since $p_{r s}$ and $q_{r s}$ are linearly independent, the planes never coincide.

The argument is divided into two cases, (i) $(\epsilon p q) \neq 0$, (ii) $(\epsilon p q)=0$.

(i) $(\epsilon p q) \neq 0$. Suppose we want, say, $p_{3^{\prime} \mathbf{4}^{\prime}}$ and $q_{1^{\prime} 2^{\prime}}$ to be the only surviving elements in the tensors. Then consider the transformation

$$
C_{8^{\prime}}^{r}=\lambda_{(s)^{\prime}}^{r}
$$

where $\lambda_{(a)^{\prime}}^{r}$ and $\lambda_{(b)^{\prime}}^{r}$ satisfy (3.2). The four vectors $\lambda_{(s)^{\prime}}^{r}$ are linearly independent since $(\epsilon p q) \neq 0$, by hypothesis. Hence $\operatorname{det} C_{\boldsymbol{s}^{\prime}}^{r} \neq 0$. Since each vector is arbitrary to within a constant factor, we can easily arrange to have $\operatorname{det} C_{s^{\prime}}^{r}>0$.

Substitution of (3.4) in (1.1) and use of (3.2) shows us immediately that the only nonzero elements in the two tensors are $p_{3^{\prime} 4^{\prime}}$ and $q_{1^{\prime} 2^{\prime}}$. For example,

$$
p_{r^{\prime} a^{\prime}}=\lambda_{\left(r^{\prime}\right)}^{m} \lambda_{\left(a^{\prime}\right)}^{n} p_{m n}=0, \text { and } q_{r^{\prime} b^{\prime}}=0 .
$$

This completes case (i). We may interpret transformation (3.4) in this manner: we choose the $x^{1^{\prime}}, x^{2^{\prime}}$ axes to lie in the $p$-plane and the $x^{3^{\prime}}, x^{4^{\prime}}$ axes to lie in the $q$-plane.

(ii) $(\epsilon p q)=0$. The above transformation becomes singular since the planes now intersect in a line. Instead let us choose our $x^{r^{\prime}}$ axes such that the $x^{1^{\prime}}$ axis lies in the $p$-plane but does not coincide with the line of intersection, the $x^{3^{\prime}}$ axis lies in the $q$-plane but does not coincide

\footnotetext{
${ }^{1}$ C. C. MacDuffee, Theory of matrices, Chelsea, p. 12.
} 
with the line of intersection, and the $x^{2^{\prime}}, x^{4^{\prime}}$ coordinate plane contains the line of intersection, but this line does not coincide with either the $x^{2^{\prime}}$ axis or the $x^{4^{\prime}}$ axis. We may write this type of transformation from $x^{r}$ coordinates to $x^{r^{\prime}}$ coordinates in the following form,

$$
C_{\mathbf{s}^{\prime}}^{r}=\mu_{{\left(\mathbf{s}^{\prime}\right)}^{r},}
$$

where the vectors $\mu_{\left(s^{\prime}\right)}^{r}$ satisfy the equations

$$
\begin{array}{ll}
p_{r s} \mu_{1}^{r}=0, & q_{r s}^{r} \mu_{3}=0, \\
p_{r s}\left(\mu_{2}^{r}+\mu_{4}^{r}\right)=0, & q_{r s}\left(\mu_{2}^{r}+\mu_{4}^{r}\right)=0, \\
p_{r s} \mu_{a}^{r} \neq 0(a=2,3,4), & q_{r s} \mu_{b}^{r} \neq 0(b=1,2,4) .
\end{array}
$$

It is clear that the vectors $\mu_{\left(s^{\prime}\right)}^{r}$, restricted only by equations (3.6), can be chosen so that they are linearly independent. Then, by multiplying say $\mu_{(1)}^{\tau}$ by a factor with a suitable sign, we shall have $\operatorname{det} C_{z^{\prime}}^{\text {' }}$ $>0$.

Substitution of (3.5) in (1.1) and use of (3.6) shows immediately that the only surviving elements in the two tensors are $p_{3^{\prime} 4^{\prime}}, p_{2^{\prime} 3^{\prime}}$, $q_{1^{\prime} 2^{\prime}}, q_{1^{\prime} \mathbf{1}^{\prime}}$. For example,

$$
\begin{aligned}
& p_{r^{\prime} 1^{\prime}}=\mu_{\left(r^{\prime}\right)}^{m} \mu_{\left(1^{\prime}\right)}^{n} p_{m n}=0, \\
& p_{2^{\prime} 4^{\prime}}=\mu_{\left(2^{\prime}\right)}^{m} \mu_{\left(4^{\prime}\right)}^{n} p_{m n}=-\mu_{\left(2^{\prime}\right) \mu_{\left(2^{\prime}\right)}^{n} p_{m n}=0,}^{n} \\
& p_{3^{\prime} 4^{\prime}}=\mu_{\left(3^{\prime}\right)}^{m} \mu_{\left(4^{\prime}\right)}^{n} p_{m n}=-\mu_{\left(3^{\prime}\right) \mu_{\left(2^{\prime}\right)}^{n} p_{m n}=p_{2^{\prime} 3^{\prime}} .}
\end{aligned}
$$

In particular we notice that $p_{3^{\prime} 4^{\prime}}=p_{2^{\prime} 3^{\prime}}$ and $q_{1^{\prime} 2^{\prime}}=q_{4^{\prime} 1^{\prime}}$.

Proof of Theorem II. There are two cases. Assume firstly $(\epsilon p q) \neq 0$. Suppose, as above, that the only nonzero elements in the two tensors are $p_{3^{\prime} 4^{\prime}}$ and $q_{1^{\prime} 2^{\prime}}$. Consider the diagonal transformation

$$
C_{s^{\prime \prime}}^{r^{\prime}}=k_{(r)} \delta_{s}^{r} \quad \text { ( } r \text { not summed). }
$$

From this we obtain $p_{3^{\prime \prime} 4^{\prime \prime}}=k_{3} k_{4} p_{3^{\prime} 4^{\prime}}$ and $q_{1^{\prime \prime} 2^{\prime \prime}}=k_{1} k_{2} q_{1^{\prime} 2^{\prime}}$. We are demanding that det $C_{z^{\prime \prime}}^{\gamma^{\prime \prime}} \equiv k_{1} k_{2} k_{3} k_{4}>0$. However $(\epsilon p q)^{\prime}$ is a tensor density and therefore transforms thus:

$$
(\epsilon p q)^{\prime \prime}=(\epsilon p q)^{\prime} \operatorname{det} C_{8^{\prime \prime}}^{r^{\prime}}
$$

That is, by (1.3), the sign of $(\epsilon p q)$ is invariant under transformations with positive determinant. Now $(\epsilon p q)^{\prime}=q_{1^{\prime} 2^{\prime}} p_{3^{\prime} 4^{\prime}}$, and so if $(\epsilon p q)>0$ we can choose $k_{1}, k_{2}, k_{3}, k_{4}$ such that $p_{3^{\prime \prime} 4^{\prime \prime}}=q_{1^{\prime \prime} 2^{\prime \prime}}$, and this is a $q$-canonical coordinate system. If $(\epsilon p q)<0$ then $k_{1}, k_{2}, k_{3}, k_{4}$ can be chosen so that $p_{3^{\prime \prime}} \mathbf{4}^{\prime \prime}=-q_{1^{\prime \prime 2} \mathbf{2}^{\prime \prime}}$ which is a $p$-canonical system. How- 
ever these two systems are equivalent to one another since we can get from a $p$-system to a $q$-system by a nonsingular transformation which does not reorient the axes. To see this consider the transformation

$$
C_{8^{\prime \prime \prime}}^{r^{\prime \prime}}=\left(\begin{array}{llll}
0 & 0 & 0 & 1 \\
0 & 0 & 1 & 0 \\
0 & 1 & 0 & 0 \\
1 & 0 & 0 & 0
\end{array}\right)
$$

applied to a $q$-canonical coordinate system in which $p_{3^{\prime \prime} 4^{\prime \prime}}=q_{1^{\prime \prime} 2^{\prime \prime}}$ are the only nonzero elements. We obtain in the new system $p_{1^{\prime \prime \prime}{ }^{\prime \prime \prime}}$ $=q_{3^{\prime \prime \prime} \prime \prime \prime \prime}$ and all other components are zero; but this is a $p$-canonical coordinate system, and $\operatorname{det} C_{s^{\prime \prime \prime}}^{r^{\prime \prime}}=1$. Similarly transformation (3.8) turns a $p$-system in which $p_{12}$ and $q_{34}$ are the only nonzero elements into a $q$-system in which $p_{34}$ and $q_{12}$ are the only nonzero elements. Thus when $(\epsilon p q) \neq 0$ we can find a transformation $C_{s^{\prime}}^{r}$, $\operatorname{det} C_{\boldsymbol{s}^{\prime}}^{\top}>0$, which will give either $p$-canonical or $q$-canonical coordinates.

Now assume $(\epsilon p q)=0$. Let us take a coordinate system in which each tensor has only two nonvanishing elements, say $p_{3^{\prime} 4^{\prime}}=p_{2^{\prime} 3^{\prime}}$ and $q_{1^{\prime} \mathbf{2}^{\prime}}=q_{4^{\prime} 1^{\prime}}$, and apply to it the diagonal transformation

$$
C_{s^{\prime \prime}}^{r^{\prime}}=l_{(r)} \delta_{s^{\prime \prime}}^{r^{\prime}}
$$

( $r$ not summed)

where $l_{(r)}=\left(p_{3^{\prime} 4^{\prime}}, l_{2} q_{1^{\prime} 2^{\prime}}, l_{4}\right)$. In the $x^{r^{\prime \prime}}$ system we then have

$$
\begin{array}{ll}
p_{3^{\prime \prime} 4^{\prime \prime}}=l_{4} q_{1^{\prime} 2^{\prime}} p_{3^{\prime} 4^{\prime}}, & q_{1^{\prime \prime} 2^{\prime \prime}}=l_{2} p_{3^{\prime} 4^{\prime}} q_{1^{\prime} 2^{\prime}}, \\
p_{2^{\prime \prime} 3^{\prime \prime}}=l_{2} q_{1^{\prime} 2^{\prime}} p_{3^{\prime} 4^{\prime}}, & q_{1^{\prime \prime} 4^{\prime \prime}}=-l_{4} p_{3^{\prime} 4^{\prime}} q_{1^{\prime} 2^{\prime}} .
\end{array}
$$

If $p_{3^{\prime} 4^{\prime}} q_{1^{\prime} 2^{\prime}}>0$ we can take $l_{2}=l_{4}$, thus making $\operatorname{det} C_{s^{\prime \prime}}^{r^{\prime}}>0$, and have a $q$-canonical system; while if $p_{3^{\prime} 4^{\prime}} q_{1^{\prime} 2^{\prime}}<0$ we can take $l_{2}=-l_{4}$ and have a $p$-canonical system. As above we can transform from a $p$-system to a $q$-system very easily. In this case apply the transformation

$$
C_{8^{\prime \prime \prime}}^{r^{\prime \prime}}=\left(\begin{array}{llll}
0 & 0 & 1 & 0 \\
0 & 0 & 0 & 1 \\
1 & 0 & 0 & 0 \\
0 & 1 & 0 & 0
\end{array}\right) .
$$

This completes the proof of Theorem II. It is now evident that for skew-symmetric tensors with vanishing determinants we can pass from any $p$-canonical coordinate system to a $q$-canonical coordinate system and vice-versa by means of a transformation of positive determinant. 
Proof of Theorem III. That equation (3.1) is a necessary condition may be seen by multiplying (2.1) by $Q_{m^{\prime} n^{\prime}}$ and (2.2) by $-P_{m^{\prime} n^{\prime}}$ and adding. Now if (3.1) holds and if the determinant of one tensor vanishes so must the determinant of the other. The case in which both determinants are zero has already been dealt with by Theorem II. We can therefore now assume that neither $(\epsilon P P)$ nor $(\epsilon Q Q)$ vanishes. We shall show the sufficiency of (3.1) by reducing the problem to the case already considered in Theorem II.

Let us assume (3.1) is satisfied at the point $A$. Consider the equation

$$
\operatorname{det}\left(P_{r s}-\theta Q_{r s}\right) \equiv\left(\epsilon P P-2 \theta \epsilon P Q+\theta^{2} \epsilon Q Q\right)^{2}=0 .
$$

Since $(\epsilon Q Q) \neq 0,(\epsilon P P) \neq 0$ we may write this, using (3.1), as

$$
\theta^{2}-2 \theta \frac{\epsilon P Q}{\epsilon Q Q}-1=0 .
$$

This quadratic equation always has two real distinct roots. Let them be $\theta_{1}$ and $-1 / \theta_{1}$. These roots are invariant under all transformations. If we now define

$$
\begin{aligned}
& p_{r s}=P_{r s}-\theta_{1} Q_{r s}, \\
& q_{r s}=\theta_{1} P_{r s}+Q_{r s},
\end{aligned}
$$

we have det $p_{r s}=\operatorname{det} q_{r s}=0$ as in Theorem II. Equations (3.12) have a tensor character and therefore remain the same in all coordinate systems. Furthermore,

$$
\begin{aligned}
(\epsilon p q) & =\left(\epsilon\left(P-\theta_{1} Q\right)\left(\theta_{1} P+Q\right)\right) \\
& =\left(1-\theta_{1}^{2}\right)(\epsilon P Q)+\theta_{1}((\epsilon P P)-(\epsilon Q Q)) ;
\end{aligned}
$$

using (3.1) and (3.11) we obtain

$$
(\epsilon p q)=\frac{2 \theta_{1}}{(\epsilon P P)}\left\{(\epsilon P P)^{2}+(\epsilon P Q)^{2}\right\} .
$$

Since we are assuming that $(\epsilon P P) \neq 0$, it follows that $(\epsilon p q) \neq 0$; moreover since we can take $\theta_{1}$ as either the positive or negative root of (3.11), we may have either $(\epsilon p q)>0$ or $(\epsilon p q)<0$ as we please. By Theorem II we can find both $p$-canonical and $q$-canonical coordinates for $p_{r s}$ and $q_{r s}$. We shall now establish the penultimate result that:

If the coordinates are $p$-canonical or $q$-canonical for $p_{r s}$ and $q_{r s}$ they are also respectively $P$-canonical or $Q$-canonical for $P_{r s}$ and $Q_{r s}$, as related by (3.12). 
Assuming the coordinates to be $p$-canonical for $p_{r s}$ and $q_{r s}$ and using (3.12) it is clear that

$$
\begin{aligned}
& \eta^{r m} \eta^{s n}\left(P_{r s}-\theta_{1} Q_{r s}\right)=\epsilon^{m n t u}\left(\theta_{1} P_{t u}+Q_{t u}\right), \\
& \eta^{r m} \eta^{s n}\left(\theta_{1} P_{r s}+Q_{r s}\right)=-\epsilon^{m n t u}\left(P_{t u}-\theta_{1} Q_{t u}\right) .
\end{aligned}
$$

Multiplying (3.15) by $\theta_{1}$ and adding that to (3.14) we see that

$$
\left(1+\theta_{1}^{2}\right) \eta^{r m} \eta^{s n} P_{r s}=\left(1+\theta_{1}^{2}\right) \epsilon^{m n t u} Q_{t u} \text {. }
$$

However $1+\theta_{1}^{2} \neq 0, \theta_{1}$ being real, and therefore the coordinates are $P$-canonical for $P_{r s}$ and $Q_{r s}$. Similarly if the coordinates are $q$-canonical for $p_{\xi s}$ and $q_{r s}$, they are $Q$-canonical for $P_{r s}$ and $Q_{r s}$. We have already seen that we may pass from a $p$-canonical coordinate system to a $q$-canonical coordinate system by a transformation of positive Jacobian. Hence we deduce at once that Theorem III is true.

I would like to thank Professor J. L. Synge for suggesting this problem which arose in connection with his paper Electromagnetism with metric, Proceedings of Symposia in Applied Mathematics, vol. 2, 1950.

Carnegie Institute of Technology 